\title{
Determination of the relative influences of carbon doping and disorder on field and temperature dependent critical current density of $\mathrm{MgB2}$
}

\begin{abstract}
$\mathrm{SiC}$ was mixed with $\mathrm{Mg}$ and $\mathrm{B}$ and reacted by either a one-step in situ or two-step method at 650 or $850^{\circ} \mathrm{C}$. By doing so, it was possible to determine the extent to which scattering via $\mathrm{C}$ doping influences the magnitude of field dependent critical current density, Jc $(\mathrm{H})$, compared to pinning via generation of microstructural disorder. The one-step reaction method leads to $\mathrm{Mg} 2 \mathrm{Si}$ formation and at the same time to more $\mathrm{C}$ doping of $\mathrm{MgB} 2$ than the two-step method. Carbon increases both the irreversibility field, Hirr, and upper critical field, Hc2 (T<28 K). However, for the temperatures $(6$ and $20 \mathrm{~K}$ ) and fields (up to $7 \mathrm{~T}$ ) studied, pinning rather than scattering overwhelmingly dominates the magnitude of the field dependent critical current density.
\end{abstract}

Keyword: Carbon doping; Microstructural disorder; MgB2 